\title{
Dynamics Analysis of Memristor Chaotic Circuit with Coexisting Hidden Attractors
}

\section{Mei Guo}

Shandong University of Science and Technology

\section{Kaixuan Zhao}

Shandong University of Science and Technology

Jiandong Liu

Shandong University of Science and Technology

\section{Xinjie Ma}

Shandong University of Science and Technology

\section{Huaying Duan}

Shandong University of Science and Technology

Gang Dou ( $\sim$ dougang521@163.com )

Shandong University of Science and Technology

\section{Research Article}

Keywords: Dynamic analysis, Memristor, Chaotic circuit, Coexisting hidden attractors

Posted Date: January 24th, 2022

DOI: https://doi.org/10.21203/rs.3.rs-1270924/v1

License: (c) (i) This work is licensed under a Creative Commons Attribution 4.0 International License.

Read Full License 


\title{
Dynamics analysis of memristor chaotic circuit with coexisting hidden attractors
}

\author{
Mei Guo · Kaixuan Zhao · Jiandong Liu · Xinjie Ma · Huaying Duan · Gang Dou
}

Received: date / Accepted: date

\begin{abstract}
In this paper, a novel $\mathrm{Sr}_{0.97} \mathrm{Ba}_{0.03} \mathrm{TiO}_{3-\delta}$ memristor based fifth-order chaotic circuit was proposed. A new cubic nonlinear magnetic control model was established by analyzing the measured data. The equilibrium points and its stability of the circuit system were analyzed by the Jacobi matrix method, and the effects of the initial states and circuit parameters on the system were discussed though methods of Lyapunov exponents spectra, bifurcation diagrams, phase diagrams, and poincaré maps. The results show that the chaotic circuit can produce complex dynamic phenomena with the variation of initial states and circuit parameters. The dynamic phenomena such as coexisting attractors have been discovered. In particular, coexisting hidden attractors were generated in the chaotic circuit, which was of great significance in engineering applications.
\end{abstract}

Keywords Dynamic analysis - Memristor - Chaotic circuit · Coexisting hidden attractors

\section{Introduction}

The first physical memristor was reported by the HewlettPackard Laboratory in 2008 [1]. It confirmed the existence of the fourth basic circuit element, that is memristor. The memristor have attracted the attention of many scholars for their potential applications in cellular neural network [2,3], associative and non-associative learning [4,5], circuit implemented electronic neurons [6], memory circuit [7], nonlinear oscillating circuits and other fields [8-11]. Because of

Mei Guo · Kaixuan Zhao · Jiandong Liu · Xinjie Ma · Huaying Duan · Gang Dou

College of Electrical Engineering and Automation,

Shandong University of Science and Technology, Qingdao 266590,

China

E-mail: dougang521@163.com the unique nonlinear characteristics of memristor, the chaotic circuit based on memristor has more abundant dynamic behaviors.

Since the chaos phenomenon was discoveried in 1963 [12], many scholars have started to study chaos theory. Chaos circuit can be used as an important way to study chaos theory. Chua proposed the Chuas circuit with a new type of strange attractor [13], which had a very simple structure and generated a variety of dynamic behaviors. Chua and Itoh proposed a new chaotic circuit which uses a memristor to replace the traditional Chua diode in Chuas circuit [14]. Some interesting dynamic phenomena were also observed in the memristor chaotic circuit [15-18]. For example, multistability is a common phenomenon in many nonlinear dynamical systems [19-25]. In Ref. [22], a new two-memristor-based chaotic circuit was proposed and the coexistence of multiple attractors was analyzed. The multistability phenomenon of memristor-based chaotic circuits extremely relies on the initial conditions and the circuit parameters, which can produce some behaviors such as coexisting attractors and coexisting limit cycle.

Another kind of attractor newly proposed in recent years was called hidden attractor, whose basin does not intersec$\mathrm{t}$ with the small neighborhood of any equilibrium points. The coexisting attractors and hidden attractors are of considerable importance in nonlinear dynamics and engineering applications [26-32]. In Ref. [30], a new three-dimensional quadratic two-wing hidden chaotic system is proposed, and fractional order multi-wing chaotic hidden attractors are generated in the system. In Ref. [31], the system has coexisting hidden attractors for some range of parameters.

In order to better apply the characteristics of physical memristor in chaotic circuits, this paper presents a fifth-order chaotic circuit based on the improved $\mathrm{Sr}_{0.97} \mathrm{Ba}_{0.03} \mathrm{TiO}_{3-\delta}$ (SBT) memristor which was prepared in our laboratory [33], and the dynamic behaviors of the circuit system were ana- 
lyzed through various methods. The rest of this paper is organized as follows. In Sect. 2, a modeling of memristor is established. In Sect. 3, The memristor chaotic circuit is designed. In Sect. 4, the stability of the system is analyzed, and the dynamics of dependence on the initial states are studied by means of numerical simulations. In particular, the coexisting hidden attractors can be found in this system. In addition, the influences of circuit parameters on dynamic behaviors of the system are studied in Sect. 5. The conclusions are given in Sect. 6.

\section{Modeling of memristor}

The $\mathrm{Sr}_{0.97} \mathrm{Ba}_{0.03} \mathrm{TiO}_{3-\delta}$ (SBT) nanoscale memristor was fabricated using magnetron sputtering technology [33]. The I-V characteristic of the SBT memristor was tested and the measured data was obtained as shown in Fig. 1, which exhibits an obvious "8" hysteresis curve. It indicates that the SBT memristor can fit the basic requirements of the memristor characteristics well. The measured current $I$ and voltage $U$ were processed using the subsection-average method [34], the average current and average voltage after pre-processing are described in red line.

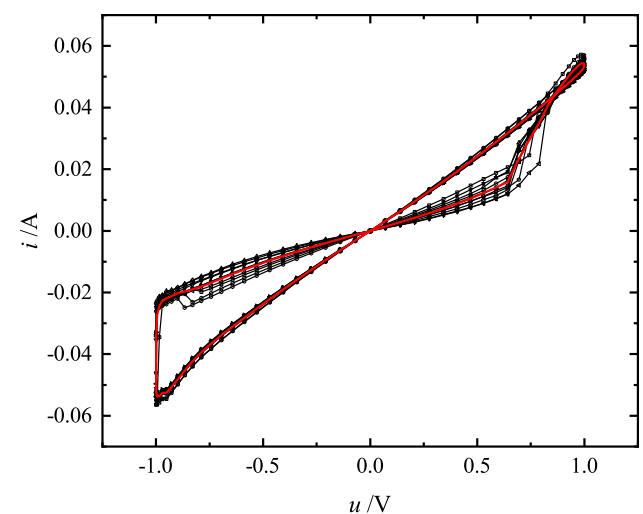

Fig. 1 The measured $I-V$ curve (black line) and measured $I-V$ curve after pre-processing of the SBT memristor(red line)

The charge $q$ and flux $\varphi$ was obtained by integrating the current and voltage data respectively, and the resulting discrete point plots of $q$ and $\varphi$ are shown in Fig. 2. The mathematical models for the memristor are commonly the cubic nonlinear model, the quadratic nonlinear model and the segmented linear model. In this paper, the cubic nonlinear model is used to fit the discrete point plots of $q$ and $\varphi$. The fitting results are shown in Fig. 2(blue line). Among them, the adopted cubic nonlinear model is

$q(\varphi)=a \varphi+b \varphi^{3}$ where $a>0, b>0$.

$u(t)=\frac{d \varphi(t)}{d t}$

$i(t)=\frac{d q(t)}{d t}$

$W(\varphi)=\frac{i(t)}{u(t)}$

Therefore, the memristive value $W(\varphi)$ of the memristor is:

$W(\varphi)=\frac{d q(\varphi)}{d \varphi}=a+3 b \varphi^{2}$

The fitting result was selected is:

$q=0.018 \varphi+0.026 \varphi^{3}$

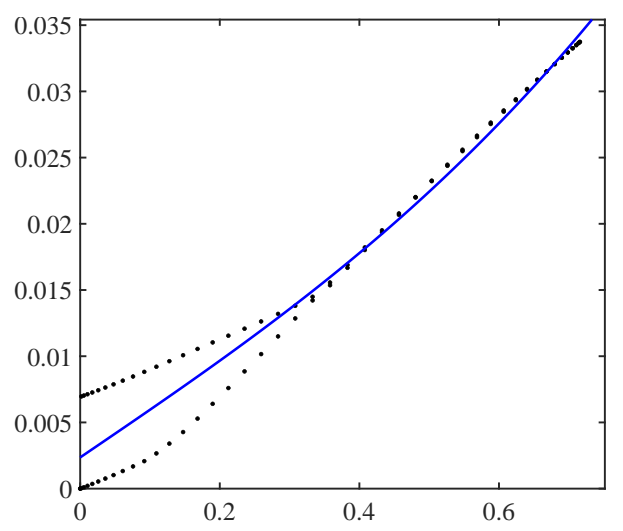

Fig. 2 Fitting curve of magnetic flux $\varphi$ and charge $q$ of SBT memristor

The value of the memristive conductance is as follows:

$W(\varphi)=0.018+3 * 0.026 \varphi^{2}$

This mathematical model for the SBT memristor was derived as:

$$
\left\{\begin{aligned}
i(t) & =W(\varphi(t)) u(t) \\
\frac{d \varphi(t)}{d t} & =u(t) \\
\frac{d q(t)}{d t} & =i(t) \\
W(\varphi(t)) & =P+Q \varphi^{2}=0.018+3 * 0.026 \varphi^{2}
\end{aligned}\right.
$$

where, $P=0.018 S, Q=3 * 0.026 S / W b^{2}, i(t)$ is the current flowing through the memristor, $u(t)$ is the voltage across the memristor, $\varphi(t)$ is the magnetic flux of the memristor, and $W$ is the memristive conductance value of the memristor. 


\section{Design of memristor chaotic circuit}

The circuit diagram of the SBT memristor chaotic circuit is depicted in Fig. 3.



Fig. 3 The chaotic circuit based on memristor

The circuit in the Fig. 3 consists of four linear capacitors $C_{1}, C_{2}, C_{3}$ and $C_{4}$, an operational amplifier $\mathrm{A}$, six linear resistors $R_{1}, R_{2}, R_{3}, R_{4}, R_{a}$ and $R_{b}$, a linear conductance $G$ and a nonlinear SBT memristor $W(\varphi)$, in which $C_{1}, C_{2}, C_{3}$, $R_{1}, R_{2}, R_{3}$ constitute the double $\mathrm{T}$ network. In this circuit, an oscillating circuit is composed of the double T network and operational amplifier A, which generate the voltage oscillating signals. When the parameters are $R_{1}=R_{2}=R, R_{3}=0.5 R$, $C_{1}=C_{2}=C, C_{3}=2 C$ and the magnification is $A=1+R_{a} / R_{b}$, the oscillation condition is satisfied and chaotic behavior may be generated. According to Ohm's law and Kirchhoff's law, the state equation of the circuit can be obtained as follows:



where $i_{M}=\left(P+Q \varphi^{2}\right)\left(A\left(v_{c 1}+v_{c 2}\right)+v_{c 3}+v_{c 4}\right), P=0.018 S$, $Q=3 * 0.026 S / W b^{2}$. When $x=v_{c 1} / v_{0}, y=v_{c 2} / v_{0}, z=v_{c 3} / v_{0}, u=$ $v_{c 4} / v_{0}, \tau=t / \tau_{0}, \tau_{0}=R_{0} C_{0}, \alpha=C_{0} / C_{4}, \beta=R_{0} / R_{4}, \gamma=C_{0} / C, \eta$ $=R_{0} / R, G_{0}=R_{0} G, P_{0}=P R_{0}, Q_{0}=Q \varphi_{0}^{2} R_{0}$, and $v_{0}=1 \mathrm{~V}, \varphi_{0}=1 \mathrm{~Wb}$, $R_{0}=1 \Omega, C_{0}=1 \mathrm{~F}, P_{0}=0.018, Q_{0}=3 * 0.026, R_{0} C_{0} v_{0} / \varphi_{0}=1$, Eq. 9 can be expressed to dimensionless dynamical system as follows:

$\left\{\begin{array}{l}\frac{d x}{d \tau}=3 \gamma \eta(A-1) x+\gamma \eta(3 A-1) y+\gamma \eta z \\ \frac{d y}{d \tau}=\gamma \eta(A-1)(x+y)+\gamma \eta z \\ \frac{d z}{d \tau}=-\frac{\gamma}{2}\left(\eta(2 A-1)(x+y)+2 \eta z+\left(f(w)+G_{0}\right)(A(x+y)+z+u)\right) \\ \frac{d u}{d \tau}=-\alpha\left(\beta u+\left(f(w)+G_{0}\right)(A(x+y)+z+u)\right) \\ \frac{d w}{d \tau}=A(x+y)+z+u\end{array}\right.$

Set the variable parameters as: $\alpha=21.5, \beta=0.667, \gamma=1, \eta$ $=1, A=1, G_{0}=-0.6, f(w)=P_{0}+Q_{0} w^{2}=0.018+3 * 0.026 w^{2}$. A double-scroll chaotic attractor is generated by MATLAB simulation under the initial condition $(0.001,0.001,0.015$, 0.001, 0.01), as shown in Fig. 4. The five Lyapunov exponents are $\mathrm{LE}_{1}=0.05875, \mathrm{LE}_{2}=0.0 .005495, \mathrm{LE}_{3}=-$ $0.001812, \mathrm{LE}_{4}=-0.8574$, and $\mathrm{LE}_{5}=-11.67$, which are calculated by using the Jacobi matrix method. The sum of Lyapunov exponents is negative, which proves that the circuit is chaotic.

\section{Dynamic analysis of the memristor circuit}

4.1 Basic dynamic analysis of the system

The right side of Eq. 10 is equal to 0, then the equilibrium point of the system can be obtained as:

$E=\{(x, y, z, u, w) \mid x=y=z=u=0, w=c\}$

where $c$ is an arbitrary constant. All the points on the $w$-axis can be an equilibrium point. The Jacobian matrix evaluated at the equilibrium point is given as:

$J=\left[\begin{array}{ccccc}3 \gamma \eta(A-1) & \gamma \eta(3 A-1) & \gamma \eta & 0 & 0 \\ \gamma \eta(A-1) & \gamma \eta(A-1) & \gamma \eta & 0 & 0 \\ -\frac{\gamma}{2}(\eta(2 A-1)+A H) & -\frac{\gamma}{2}(\eta(2 A-1)+A H) & -\gamma \eta-\frac{\gamma}{2} H & -\frac{\gamma}{2} H & 0 \\ -\alpha A H & -\alpha A H & -\alpha H & -\alpha \beta-\alpha H & 0 \\ A & A & 1 & 1 & 0\end{array}\right]$

where $H=W+G_{0}, W=0.018+3 * 0.026 c^{2}, \alpha=21.5, \beta=$ $0.667, \gamma=1, \eta=1, A=1, G_{0}=-0.6$. The characteristic equation is consequently written as:

$|\lambda E-J|=\lambda\left(\lambda^{4}+a_{1} \lambda^{3}+a_{2} \lambda^{2}+a_{3} \lambda+a_{4}\right)=0$

The parameter $w(c)=0.018+3 * 0.026 c^{2}, \alpha=21.5, \beta=$ $0.667, \gamma=1, \eta=1, A=1, G_{0}=-0.6$ in Eq. 13 :

$\left\{\begin{array}{l}a_{1}=1.716 c^{2}+2.5365 \\ a_{2}=2.3143 c^{2}-1.9276 \\ a_{3}=2.8736 c^{2}-6.1007 \\ a_{4}=2.7956 c^{2}-6.5186\end{array}\right.$

According to the Rouse stability criterion, if the roots of the Eq. 14 satisfy that all the real parts of the real and complex roots are negative, the necessary and sufficient conditions are:

$H_{k}=\left|\begin{array}{cccc}a_{1} & a_{3} & 0 & 0 \\ 1 & a_{2} & a_{4} & 0 \\ 0 & a_{1} & a_{3} & 0 \\ 0 & 1 & a_{2} & a_{4}\end{array}\right|>0$

where

$\left\{\begin{array}{l}H_{1}=a_{1}>0 \\ H_{2}=a_{1} a_{2}-a_{3}>0 \\ H_{3}=a_{1}\left(a_{2} a_{3}-a_{1} a_{4}\right)-a_{3}^{2}>0 \\ H_{4}=a_{4} H_{3}>0\end{array}\right.$ 


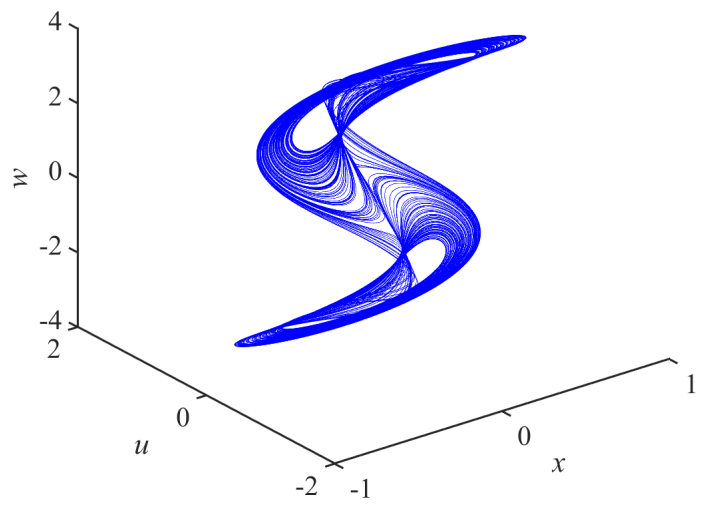

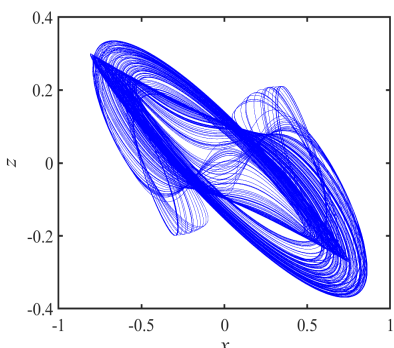

(a)

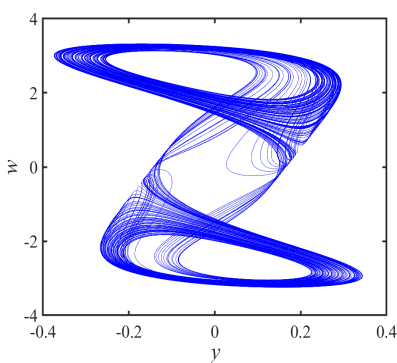

(c)

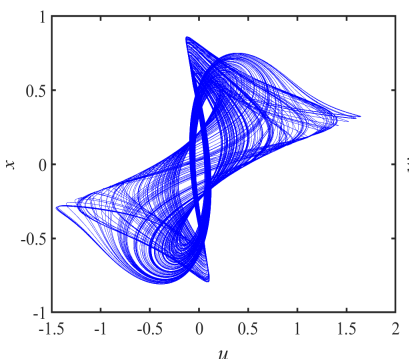

(e)

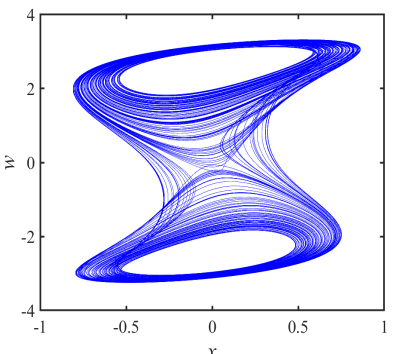

(b)



(d)

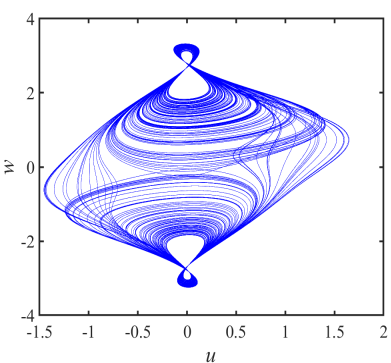

(f)
Fig. 4 Double scroll chaotic attractors of memristor chaotic system
By calculation, when $|c|>1.527$, the system is in stable state, while the $|c|<1.527$, the system equilibrium point is in an unstable state. And the system may exhibit chaos, limit cycle and other nonlinear dynamics behaviors near these unstable equilibrium points.

4.2 Effects of initial states on the dynamic characteristics of the system

\subsubsection{Dynamic analysis of dependence on the initial state $w(0)$}

When $\alpha=21.5, \beta=0.667, \gamma=1, \eta=1, A=1, G_{0}=-0.6$, the initial state is $(0.001,0.001,0.015,0.001, w(0))$. The variation range of $w(0)$ is from -5 to 5 . The Lyapunov exponents spectrum and bifurcation diagram of state variable $w$ is shown in Fig. 5. In order to see the changes of Lyapunov exponents more clearly, the four Lyapunov exponent are shown. The Lyapunov exponents spectrum of the system is highly consistent with the bifurcation diagram. With the increase of the initial state $w(0)$, the system presents a variety of dynamic behaviors. In the interval $[-5.00,-2.89]$ and $[2.84,5.00]$, the system was in stable point state. In the interval [2.88, 2.64], $[2.42,0.49],[0.55,2.32]$ and $[2.63,2.83]$, the system was in sink state. In the interval $[-0.48,-0.43]$, the system was in limit cycles state. In the interval [-2.63, -2.51], [-0.24, 0.41] and $[2.44,2.62]$, the system was in double scroll chaotic attractor state. In the intervals $[-2.50,-2.43],[-0.42,-0.25]$, $[0.42,0.54]$ and $[2.33,2.43]$, the system was in single scroll chaotic attractor state. The phase diagram of the initial state $w(0)$ in the $x-w$ plane at different special values are shown in Fig. 6. Fig. 6(a) is the limit cycle, Fig. 6(b) is the double scroll chaotic attractors, Fig. 6(c) is the single scroll chaotic attractor, Fig. 6(d) is the sinks.

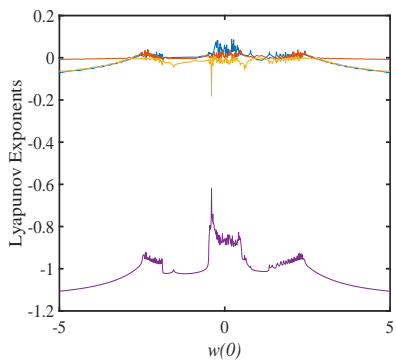

(a)

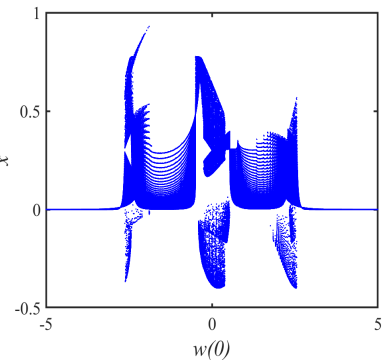

(b)
Fig. 5 a Lyapunov exponents spectrum and b bifurcation diagram varying with the initial state $w(0)$

The dynamic behavior of the initial state $w(0)$ of this chaotic system has coexistence behavior. For example, when the initial state is $(0.001,0.001,0.015,0.001, \pm 0.45)$, the 


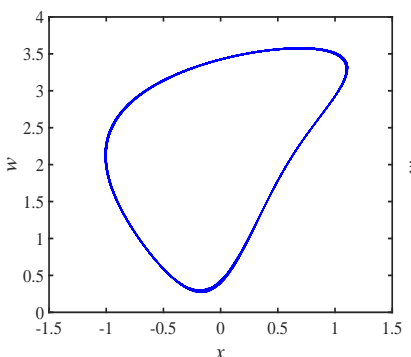

(a)

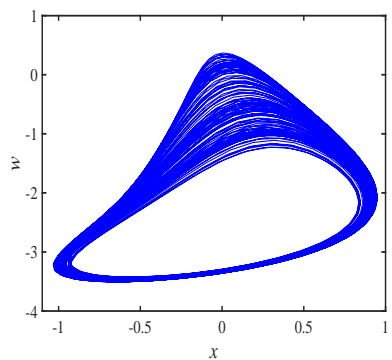

(c)

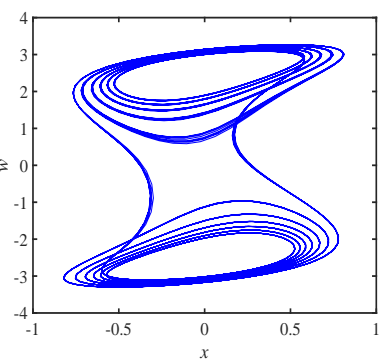

(b)

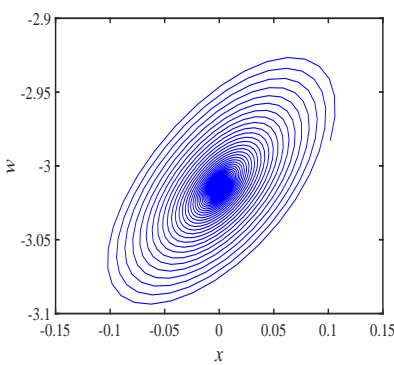

(d)
Fig. 6 The phase diagram of the initial state $w(0)$ in the $x-w$ plane at different special values; $\mathbf{a}$ the phase diagram when $w(0)=-0.46$; $\mathbf{b}$ the phase diagram when $w(0)=0.1$; $\mathbf{c}$ the phase diagram when $w(0)=0.45$; d the phase diagram when $w(0)=1.25$

system has the coexistence of single scroll chaotic attractor and limit cycles, the phase diagram and Poincaré section are shown in Fig. 7. When the initial state is $(0.001,0.001$, $0.015,0.001, \pm 0.2)$, the system has the coexistence of double scroll chaotic attractors, its phase diagram and Poincaré section are shown in Fig. 8.

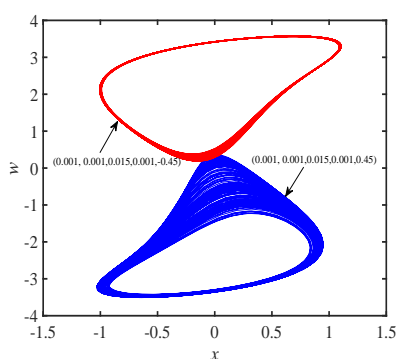

(a)

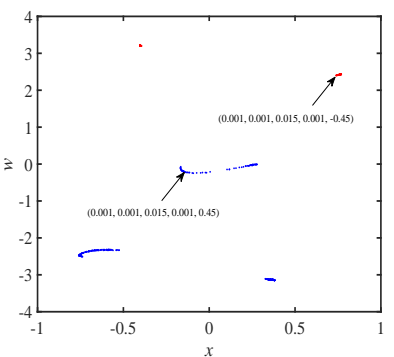

(b)
Fig. 7 Phase diagram of single scroll chaotic attractor coexisting with limit cycle and its Poincaré section

\subsubsection{Dynamic analysis of dependence on the initial state $y(0)$}

When $y(0)$ is taken as a state variable, and the other parameters of the system remain unchanged. The initial state is



(a)

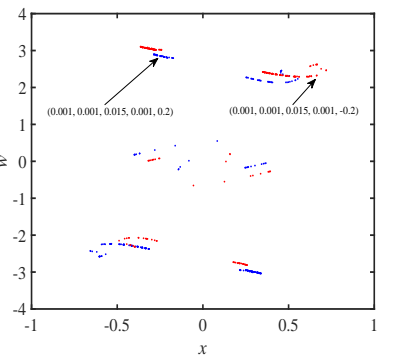

(b)
Fig. 8 Phase diagram of coexisting double scroll chaotic attractors and its Poincaré section

set to $(0.001, y(0), 0.015,0.001,0.01)$, the dynamic characteristics of $y(0)$ in the interval of $[-0.5,0.5]$ are analyzed. The Lyapunov exponents spectrum and bifurcation diagram of state variable $y$ is shown in Fig. 9. The Lyapunov exponents spectrum of the system is highly consistent with the bifurcation diagram. When the system parameters remain unchanged and the initial state is $(0.001, \pm 0.298,0.015$, $0.001,0.01)$, the coexistence of limit cycles exists in the system. The coexistence phase diagram and Poincaré section diagram are shown in Fig. 10. When the initial state is $(0.001, \pm 0.167,0.015,0.001,0.01)$, the coexistence of single scroll chaotic attractors exists in the system. The coexistence phase diagram and Poincaré section diagram are shown in Fig. 11. When the initial state is $(0.001, \pm 0.142$, $0.015,0.001,0.01)$, the coexistence of the single scroll chaotic attractor and the double scroll chaotic attractor exists in the system. The coexistence phase diagram and the Poincaré section diagram are shown in Fig. 12. When the initial state is $(0.001, \pm 0.036,0.015,0.001,0.01)$, there is a phenomenon of coexistence of double chaotic attractors in the system. The coexistence phase diagram and the Poincaré section diagram are shown in Fig. 13.

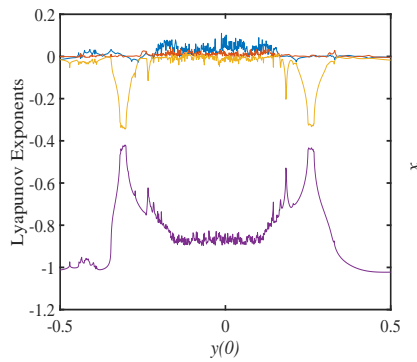

(a)

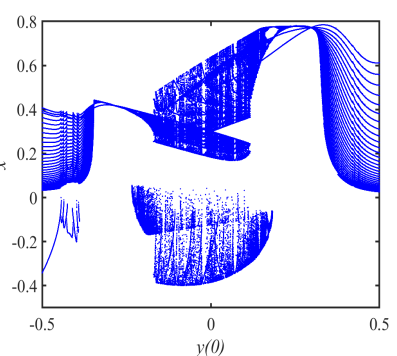

(b)
Fig. 9 a Lyapunov exponents spectrum and b bifurcation diagram varying with the initial state $y(0)$ 


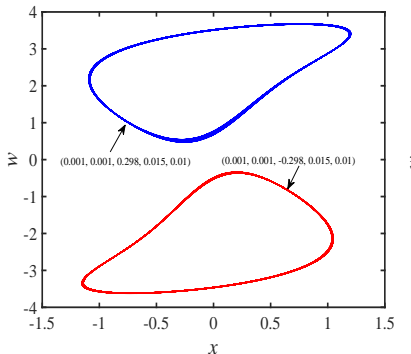

(a)

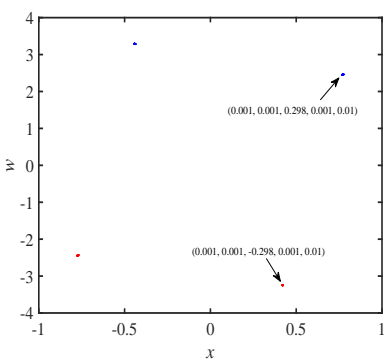

(b)
Fig. 10 Phase diagram of coexisting limit cycles and its Poincaré section

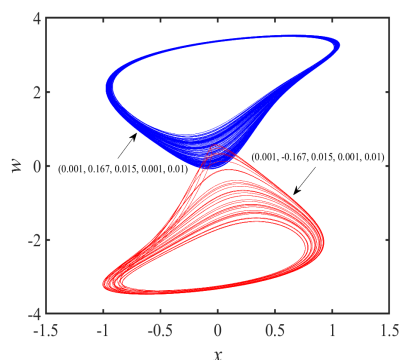

(a)

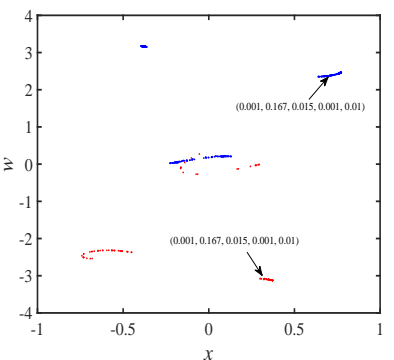

(b)
Fig. 11 Phase diagram of coexisting single scroll chaotic attractors and its Poincaré section

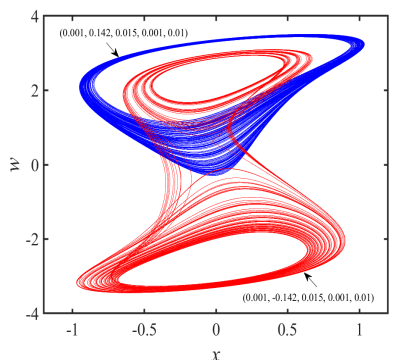

(a)

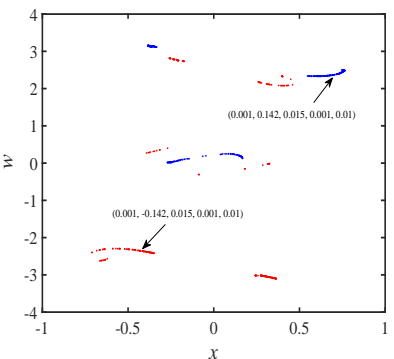

(b)
Fig. 12 Phase diagram of double scroll chaotic attractor coexisting with single scroll chaotic attractor and its Poincaré section

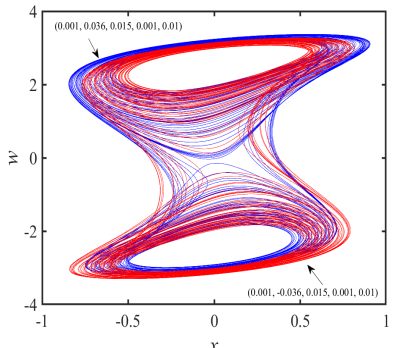

(a)

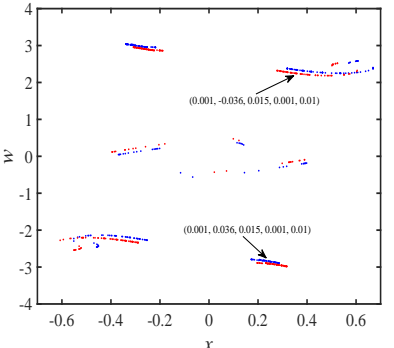

(b)
Fig. 13 Phase diagram of coexisting double scroll chaotic attractors and its Poincaré section
4.2.3 Dynamic analysis of dependence on the initial state $x(0), z(0), u(0)$

When the circuit element parameters are selected as $\alpha=21.5$, $\beta=0.667, \gamma=1, \eta=1, A=1, G_{0}=-0.6$, the dynamic characteristics of the initial state $x(0), z(0)$ and $u(0)$ in the different interval are analyzed. The corresponding Lyapunov exponents diagrams and bifurcation diagrams are displayed in Figs. 14, 15 and 16.The minimum Lyapunov exponents are not depicted for clarity.

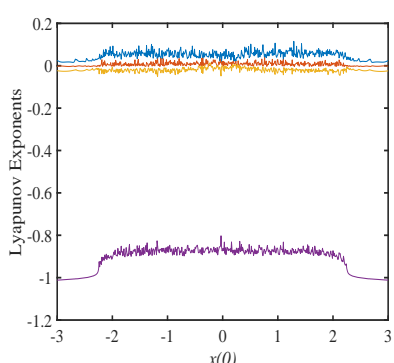

(a)

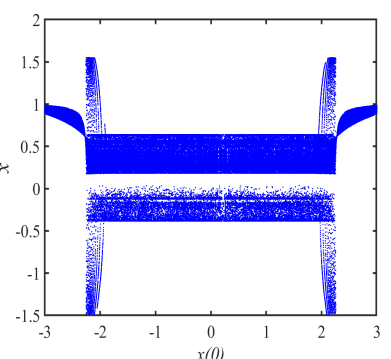

(b)
Fig. 14 a Lyapunov exponents spectrum and b bifurcation diagram varying with the initial state $x(0)$

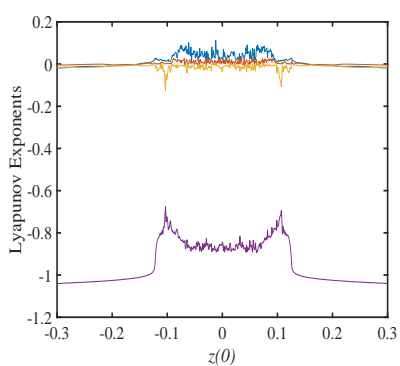

(a)

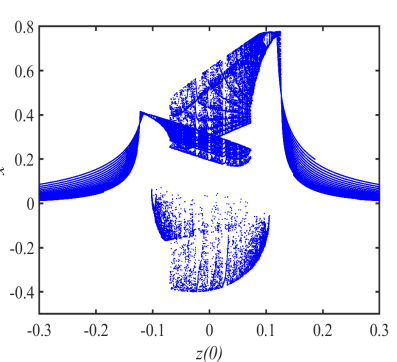

(b)
Fig. 15 a Lyapunov exponents spectrum and b bifurcation diagram varying with the initial state $z(0)$

When the initial state $x(0), z(0)$ and $u(0)$ values are changed, the chaotic system will produce the coexistence phenomenon. The initial state parameters and the coexistence behavior are shown in Table 1, and the phase diagram and the Poincaré section diagram of the coexistence phenomenon are shown in Figs. 17, 18, 19, 20, 21 and 22. 


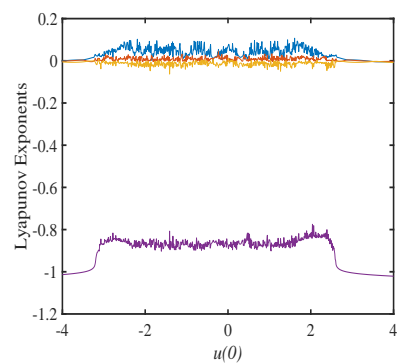

(a)

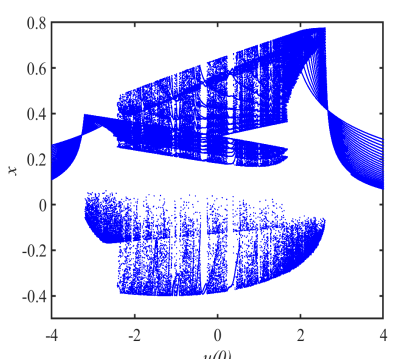

(b)

Fig. 16 a Lyapunov exponents spectrum and b bifurcation diagram varying with the initial state $u(0)$

Table 1. Coexistence behavior under different initial states

\begin{tabular}{|c|c|c|}
\hline Depicted figures & Initial conditions & Coexistence phenomenon \\
\hline Fig. 17 & $( \pm 3,0.001,0.015,0.001,0.01)$ & Limit cycles \\
\hline Fig. 18 & $( \pm 1.5,0.001,0.015,0.001,0.01)$ & $\begin{array}{c}\text { Double-scroll chaotic } \\
\text { attractors }\end{array}$ \\
\hline Fig. 19 & $(0.001,0.001, \pm 0.08,0.001,0.01)$ & $\begin{array}{l}\text { Single-scroll chaotic } \\
\text { attractors }\end{array}$ \\
\hline Fig. 20 & $(0.001,0.001, \pm 0.015,0.001,0.01)$ & $\begin{array}{c}\text { Double-scroll chaotic } \\
\text { attractors }\end{array}$ \\
\hline Fig. 21 & $(0.001,0.001,0.015, \pm 1.8,0.01)$ & $\begin{array}{c}\text { Double-scroll chaotic } \\
\text { attractors and } \\
\text { single-scroll chaotic } \\
\text { attractors }\end{array}$ \\
\hline Fig. 22 & $(0.001,0.001,0.015, \pm 0.5,0.01)$ & $\begin{array}{l}\text { Double-scroll chaotic } \\
\text { attractors }\end{array}$ \\
\hline
\end{tabular}

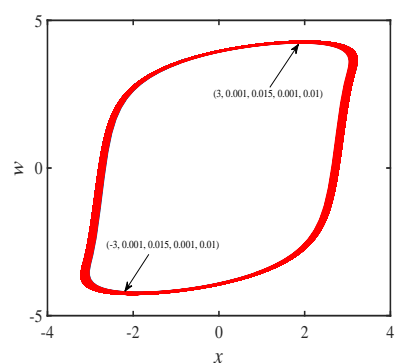

(a)

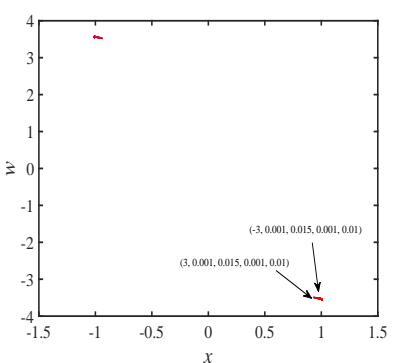

(b)

Fig. 17 Phase diagram of coexisting limit cycles and its Poincaré section

\subsection{The coexisting hidden attractors}

The hidden attractors are difficult to find because both basin$s$ of attraction and the dimension of the attractor could be very small. Coexisting hidden attractors are coexistence of multiple hidden attractors, which was called hidden multistability. At present, there are few studies on the existence of coexisting hidden attractors in actual chaotic circuits. When

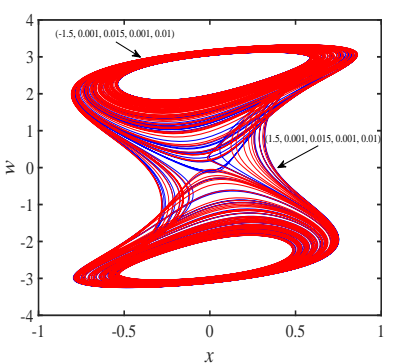

(a)

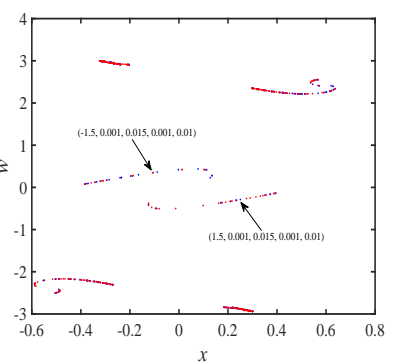

(b)
Fig. 18 Phase diagram of coexisting double scroll chaotic attractors and its Poincaré section

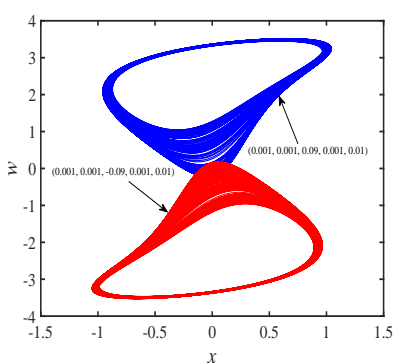

(a)

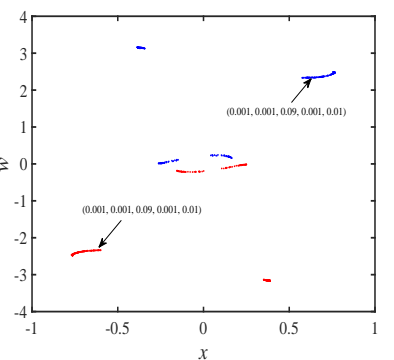

(b)
Fig. 19 Phase diagram of coexisting single scroll chaotic attractors and its Poincaré section

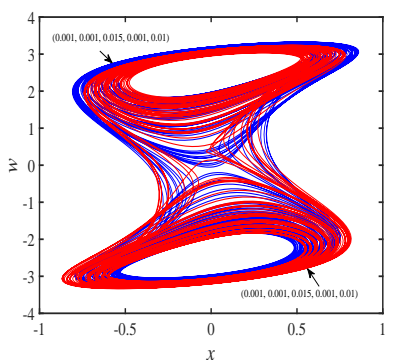

(a)

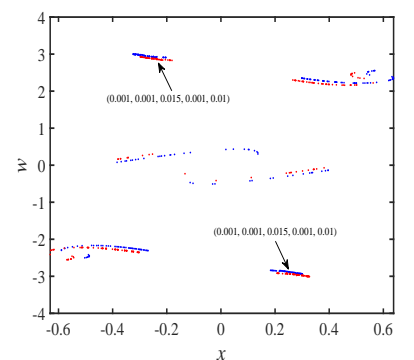

(b)
Fig. 20 Phase diagram of coexisting double scroll chaotic attractors and its Poincaré section

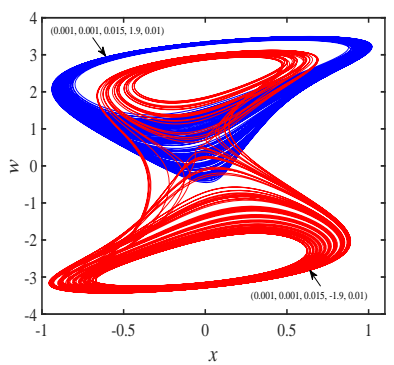

(a)

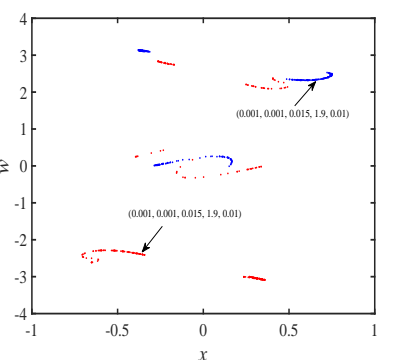

(b)
Fig. 21 Phase diagram of double scroll chaotic attractor coexisting with single scroll chaotic attractor and its Poincaré section 


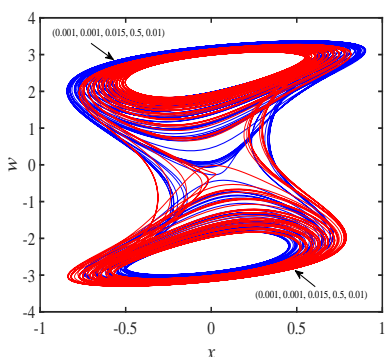

(a)

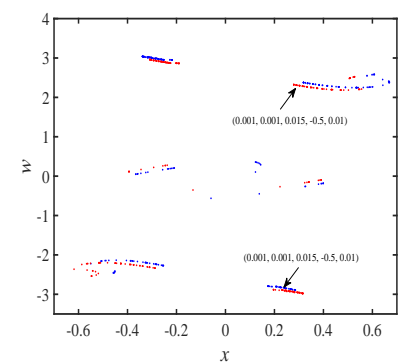

(b)
Fig. 22 Phase diagram of coexisting double scroll chaotic attractors and its Poincaré section

$-2.63<c<-2.43$ or $2.33<c<2.62$, the theoretical analysis in Sect. 4.1 shows that the equilibrium point is stable, the numerical simulation in Sect. 4.2.1 shows that the system is chaotic (Fig. 5), so the coexisting hidden attractors in blue line may exist in the system. For example, when $w(0)=2.5$, the double scroll chaotic attractor is obtained (Fig. 25). The finite-time local Lyapunov exponents on the time interval $t \in$ $[0,50]$ with $w(0)=2.5$ are shown in Fig. 23, and they are calculated as $\mathrm{LE}_{1}=0.0128, \mathrm{LE}_{2}=0.0199, \mathrm{LE}_{3}=-0.0176, \mathrm{LE}_{4}=$ $-1.0213, \mathrm{LE}_{5}=-12.6051$, which indicates that the memristor circuit system is chaotic. When $w(0)=-2.5$, the single scroll chaotic attractor in red line is obtained (Fig. 25). The finitetime local Lyapunov exponents on the time interval $t \in[0$, 50] with $w(0)=-2.5$ are shown in Fig. 24, and they are calculated as $\mathrm{LE}_{1}=0.0152, \mathrm{LE}_{2}=0.0337, \mathrm{LE}_{3}=-0.0203, \mathrm{LE}_{4}=$ $-1.0099, \mathrm{LE}_{5}=-11.9117$, which indicates that the memristor circuit system is chaotic. Consequently, the coexisting hidden chaotic attractors exist in the system.
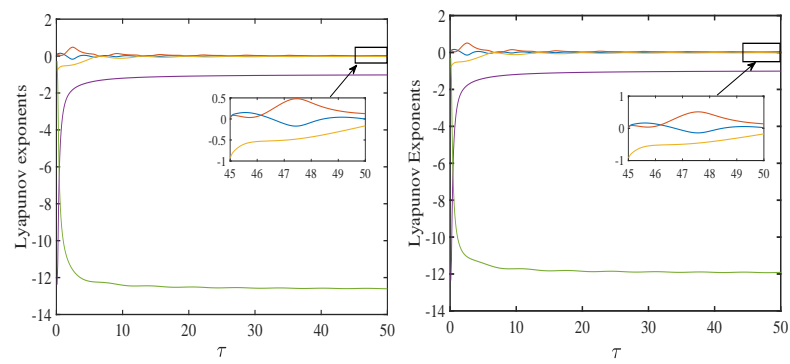

Fig. 23 Lyapunov exponents di-Fig. 24 Lyapunov exponents diagram at $w(0)=2.5$

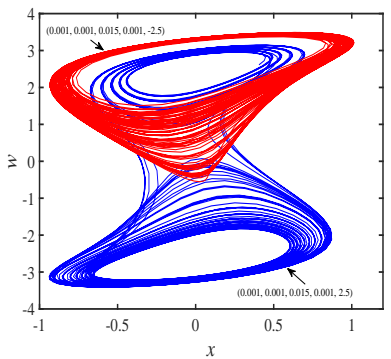

(a)

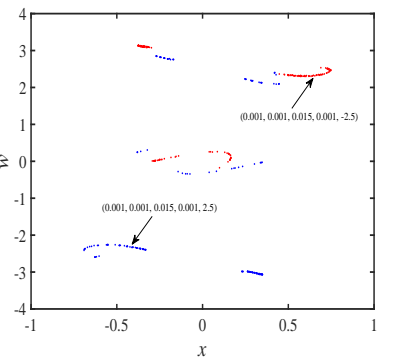

(b)
Fig. 25 Phase diagram of coexisting hidden attractors and its Poincaré section

\section{Effects of circuit parameters on the dynamic characteristics of the system}

\subsection{Influences of parameter $\alpha$ on system dynamic} behaviors

When parameter $\alpha$ is in the range of $[0,35]$ and initial values are $(0.001,0.001,0.015,0.001,0.01)$, the Lyapunov exponents spectrum and bifurcation diagram can be obtained in Fig. 26. In order to better observe the Lyapunov exponents of the chaos, the fifth Lyapunov exponential curve is not drawn. When $\alpha=5$, the system exhibits limit cycle; when $\alpha=14$, the system exhibits single scroll chaotic attractor; when $\alpha=30$, the system exhibits double scroll chaotic attractor. The phase diagram and Poincare section diagram of the dynamic behaviors are shown in Figs. 27, 28 and 29.

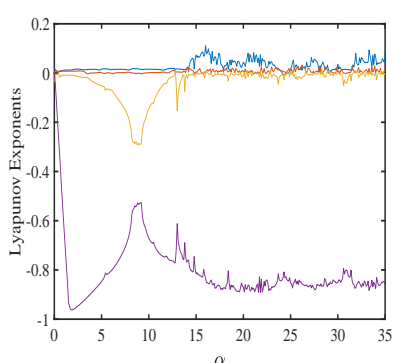

(a)

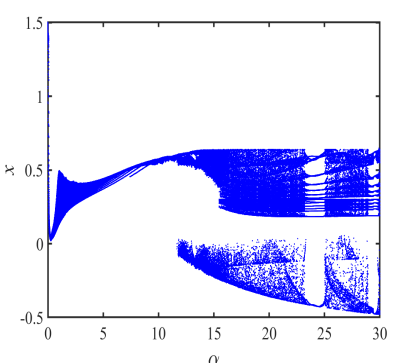

(b)
Fig. 26 Dynamic behavior of circuit parameter $\alpha$ a Lyapunov exponents spectrum and $\mathbf{b}$ bifurcation diagram

5.2 Influences of other parameters on system dynamic behaviors

The initial states are set as $(0.001,0.001,0.015,0.001,0.01)$, and the dynamics of the circuit system with the variation in the circuit parameters $\beta, \gamma$ and $\eta$, and are depicted in 


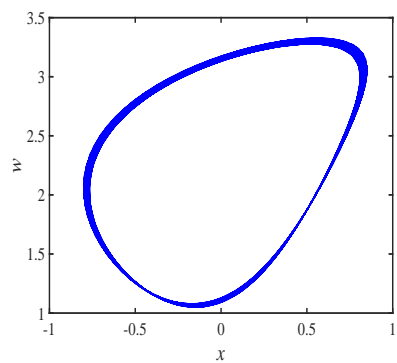

(a)

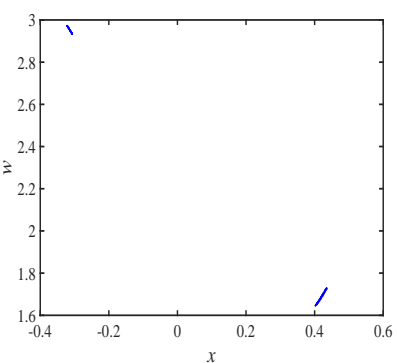

(b)
Fig. 27 When $\alpha=5$, limit cycle phase diagram and its Poincaré section

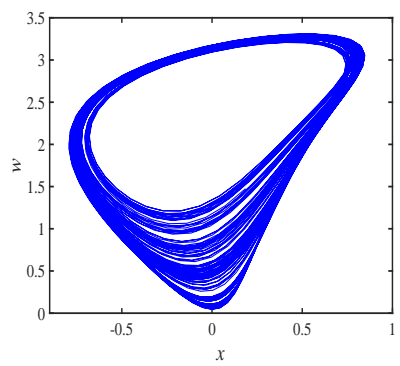

(a)

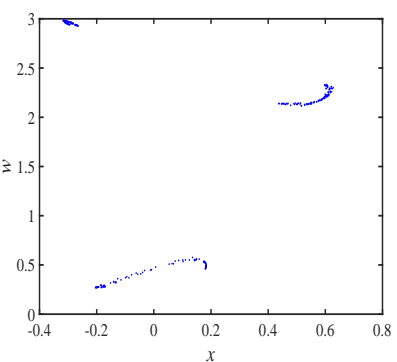

(b)
Fig. 28 When $\alpha=14$, phase diagram of single scroll chaotic attractor and its Poincaré section

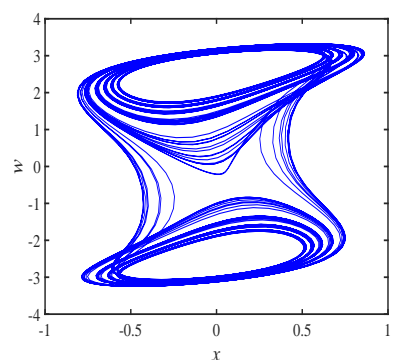

(a)

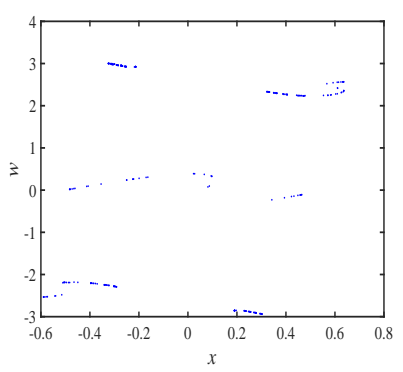

(b)
Fig. 29 When $\alpha=30$, phase diagram of double scroll chaotic attractor and its Poincaré section

Table 2. The corresponding Lyapunov exponents diagrams and bifurcation diagrams are displayed in Figs. 30, 31 and 32, the minimum Lyapunov exponents are not depicted for clarity.
Table 2. Dynamic behaviors of the circuit parameters $\beta, \gamma$ and $\eta$ in different intervals

\begin{tabular}{ccc}
\hline The circuit parameter & Interval & Dynamics \\
\hline$\beta$ & $(0.00,0.62)$ & Single-scroll attractors \\
& $(0.63,0.82)$ & Double-scroll attractors \\
$\gamma$ & $(0.83,2.00)$ & Limit cycle \\
& $(0.00,1.44)$ & Double-scroll attractors \\
& $(1.45,2.08)$ & Single-scroll attractors \\
$\eta$ & $(2.09,10.00)$ & Limit cycle \\
& $(0.00,0.87)$ & Sink \\
& $(0.88,1.44)$ & Double-scroll attractors \\
& $(1.45,1.62)$ & Single-scroll attractors \\
& $(1.63,2.00)$ & Limit cycle \\
\hline
\end{tabular}

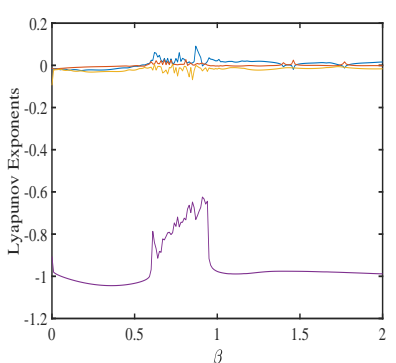

(a)

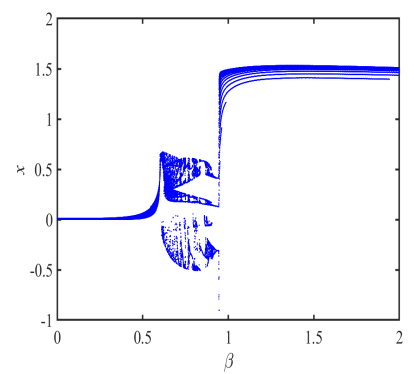

(b)
Fig. 30 Dynamic behavior of circuit parameter $\beta$ a Lyapunov exponents spectrum and $\mathbf{b}$ bifurcation diagram

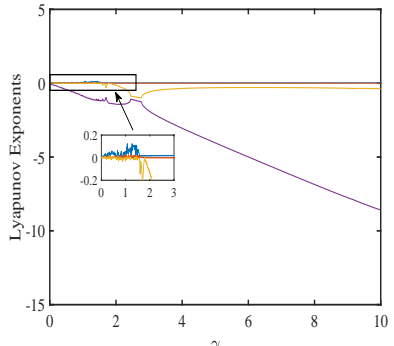

(a)

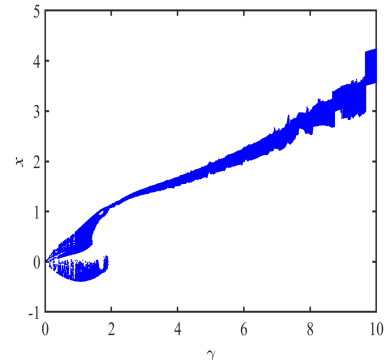

(b)
Fig. 31 Dynamic behavior of circuit parameter $\gamma$ a Lyapunov exponents spectrum and $\mathbf{b}$ bifurcation diagram

\section{Conclusion}

In the paper, the cubic memristor model of SBT memristor is established, and the single-memristor fifth-order chaotic circuit is designed. The equilibrium stability of the circuit is analyzed by numerical calculation method, and the dynamic behaviors of the system with the initial states and circuit 


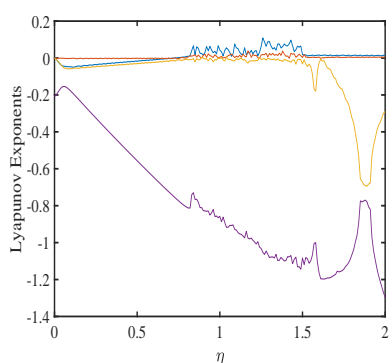

(a)

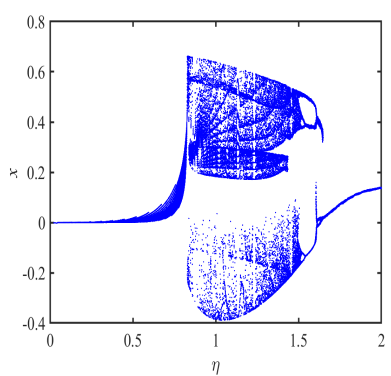

(b)
Fig. 32 Dynamic behavior of circuit parameter $\eta$ a Lyapunov exponents spectrum and $\mathbf{b}$ bifurcation diagram

parameters is studied by using phase diagram, Lyapunov exponents spectra, bifurcation diagrams and Poincaré maps. The results show that the system exhibits complex dynamic behaviors such as stable points, chaotic attractors, limit cycles and a variety of coexistence phenomena. Particularly, the system has a special dynamic behavior of coexisting hidden attractors. All the results will contribute to the practical application of chaotic circuits.

Acknowledgements This work was supported by the National Natural Science Foundation of China (Grant Nos. 62176143, 61703246, 61703247), the Natural Science Foundation of Shandong Province (ZR 2021MF001), the Talented Young Teachers Training Program of Shandong University of Science and Technology, and the Elite Project of Shandong University of Science and Technology.

\section{Compliance with ethical standards}

Conflict of interest The authors declare that they have no conflict of interest.

\section{References}

1. Strukov, D.B., Snider, G.S., Stewart, D.R., Williams, R.S.: The missing memristor found. Nature 453, 80-83 (2008)

2. Hu, X.F., Feng, G., Duan, S.K., Liu, L.: A memristive multilayer cellular neural network with applications to image processing. IEEE Trans. Neural Netw. Learn Syst. 28, 1889-1901 (2017)

3. Duan, S.K., Hu, X.F., Dong, Z.K., Wang, L.D., Mazumder, P.: Memristor-based cellular nonlinear/neural network: Design, analysis, and applications. IEEE Trans. Neural Netw. Learn Syst. 26, 1202-1213 (2015)

4. Hong, Q., Yan, R., Wang, C., Sun, J.R.: Memristive circuit implementation of biological nonassociative learning mechanism and its applications. IEEE Trans. Biomed. Circuits Syst. 14, 1036-1050 (2020)

5. Guo, M., Zhu, Y.L., Liu, R.Y., Zhao, K.X., Dou, G.: An associative memory circuit based on physical memristors. Neurocomputing 47, 12-23 (2022)

6. Li, Z.J., Zhou, H.Y.: Regulation of firing rhythms in a fourstablememristor-based HindmarshCRose neuron. Electron. Lett. 57, 715-717 (2021)
7. Guo, M., Liu, R.Y., Dou, M.L., Dou, G.: SBT-memristor-based crossbar memory circuit. Chin. Phys. B 30, 68402-068402 (2021)

8. Ma, X.J., Mou, J., Liu, J., Ma, C.G., Yang, F.F., Zhao, X.: A novel simple chaotic circuit based on memristorCmemcapacitor. Nonlinear Dyn. 100, 2859-2876 (2020)

9. Yang, Y., Li, D.D., Wang, D.Q.: Dynamic analysis of the Switched-Inductor Buck-Boost converter based on the memristor. Electronics 10, 452 (2021)

10. Wu, H., Zhou, M., Chen, M., Xu, Q., Bao, B.: DC-offset induced asymmetry in memristive diode-bridge-based Shinriki oscillator. Chaos, Solitons and Fractals 154, 111624 (2022)

11. Ye, X.L., Mou, J., Luo, C.F., Yang, F.F., Cao, Y.H.: Complexity analysis of a mixed memristive chaotic circuit. Complexity 2018, $1-9$ (2018)

12. Lorenz, E.N.: Deterministic nonperiodic flow. Journal of the Atmospheric Science 20, 130-141 (1963)

13. Belykh, V.N., Chua, L.O.: A new type of strange attractor related to the chuas circuit. J. Circuit Syst. Comp. 3, 361-374 (1993)

14. Itoh, M., Chua, L.O.: Memristor oscillators. Int. J. Bifurc. Chaos $18,3183-3206(2008)$

15. Minati, L., Gambuzza, L.V., Thio, W.J., Sprott, J.C., Frasca, M.: A chaotic circuit based on a physical memristor. Chaos Soliton. Fract. 138, $109990(2020)$

16. Lin, H.R., Wang, C.H., Tan, Y.M.: Coexisting multi-dynamics of a physical SBT memristor-based chaotic circuit. Int. J. Bifurc. Chaos 30, 2030043 (2020)

17. Guo, M., Gao, Z.H., Xue, Y.B., Dou, G., Li, Y.X.: Dynamics of a physical SBT memristor-based wien-bridge circuit. Nonlinear Dyn. 93, 1681-1693 (2018)

18. Ye, X.L., Wang, X.Y., Gao, S., Mou, J., Wang, Z.S., Yang, F.F.: A new chaotic circuit with multiple memristors and its application in image encryption. Nonlinear Dyn. 99, 1489-1506 (2020)

19. Ma, C.G., Mou, J., Xiong, L., Banerjee, S., Liu, T.M., Han, X.T.: Behavior of Multiple Memristor Circuits. Handbook of Memristor Networks, pp. 913-940 (2019)

20. Guo, M., Yang, R., Zhang, M., Liu, R.Y., Zhu, Y.L.: A novel memcapacitor and its application in a chaotic circuit. Nonlinear Dyn. 105, 877-886 (2021)

21. Bao, B.C., Jiang, T., Wang, G.Y., Jin, P.P., Bao, H., Chen, M.: Twomemristor-based Chuas hyperchaotic circuit with plane equilibrium and its extreme multistability. Nonlinear Dyn. 89, 1157-1171 (2017)

22. Guo, M., Zhang, M., Dou, M.L., Dou, G., Li, Y.X.: Dynamics of the two-SBT-memristor-based chaotic circuit. Chin. Phys. B 29, 110505 (2020)

23. Chen, M., Sun, M.X., Bao, B.C., Wu, H.G., Xu, Q., Wang, J.: Controlling extreme multistability of memristor emulator-based dynamical circuit in fluxCcharge domain. Nonlinear Dyn. 91, 13951412 (2018)

24. Guo, M., Yang, W.Y., Xue, Y.B., Gao, Z.H., Yuan, F., Dou, G., Li, Y.X.: Multistability in a physical memristor-based modified Chua's circuit. Chaos 29, 043114 (2019)

25. Huang, L.L., Wang, Y.L., Jiang, Y.C., Lei, T.F.: A novel memristor chaotic system with a hidden attractor and multistability and its implementation in a circuit. Math. Probl. Eng. 2021, 1-16 (2021)

26. Vaidyanathan, S., Sambas, A., Azar, A.T., Rana K.P.S., Kumar, V.: A new 5-D hyperchaotic four-wing system with multistability and hidden attractor, its backstepping control, and circuit simulation. Backstepping Control of Nonlinear Dynamical Systems, pp. 115138 (2021)

27. Delgado-Aranda, F., Campos-Canton, I., Tristan-Hernandez, E., Salas-Castro, P.: Hidden attractors from the switching linear systems. Sociedad Mexicana de Fłsica 66, 683-691 (2020)

28. Wang, N., Zhang, G.S., Kuznetsov, N.V., Bao, H.: Hidden attractors and multistability in a modified Chuas circuit. Commun Nonlinear Sci Numer Simul 92, 105494 (2020) 
29. Zhang, S., Zeng, Y.C.: A simple Jerk-like system without equilibrium: Asymmetric coexisting hidden attractors, bursting oscillation and double full Feigenbaum remerging trees. Chaos Solitons Fractals 120, 25-40 (2019)

30. Cui, L., Lu, M., Ou, Q.L., Duan, H., Luo, W.H.: Analysis and circuit implementation of fractional order multi-wing hidden attractors. Chaos Solitons Fractals 138, 109894 (2020)

31. Shahzad, M., Pham, V.T., Ahmad, M.A., Jafari, S.,Hadaeghi, F.: Synchronization and circuit design of a chaotic system with coexisting hidden attractors. The European Physical Journal Special Topics 224, 1637-1140 (2015)

32. Feng, Y., Rajagopal, K., Khalaf, A.J.M., Alsaadi, F.E., Alsaadi, F.E., Pham, V.-T.: A new hidden attractor hyperchaotic memristor oscillator with a line of equilibria. The European Physical Journal Special Topics 229, 1279-1288 (2020)

33. Dou, G., Yu, Y., Guo, M., Zhang, Y.M., Sun, Z., Li, Y.X.: Memristive behavior based on Ba-doped $\mathrm{SrTiO}_{3}$ films. Chin. Phys. Lett. 34, 038502 (2017)

34. Zhang, Y.M., Dou, G., Sun, Z., Guo, M., Li, Y.X.: Establishment of physical and mathematical models for $\mathrm{Sr}_{0.95} \mathrm{Ba}_{0.05} \mathrm{TiO}_{3}$ memristor. Int. J. Bifurc. Chaos 27, 1750148 (2017) 


\section{Supplementary Files}

This is a list of supplementary files associated with this preprint. Click to download.

- dataavailabilitystatement.pdf 